\title{
Erratum
}

Numer. Math. 58, 839-853 (1991)

\section{On the convergence of multistep methods for nonlinear stiff differential equations}

\section{Lubich}

Institut für Mathematik und Geometrie, Universität Innsbruck, Technikerstrasse 13, A-6020 Innsbruck, Austria

Received October 9, 1989

In the way it is stated, Theorem 4 of [4] on the convergence of multistep methods for a class of semilinear parabolic equations does not apply to NavierStokes equations as the discussion preceding that theorem erroneously suggests: The local Lipschitz continuity of the nonlinearity is not fulfilled in the way required there. The following rather straightforward generalization of Theorem 4 does however apply. See Fujita and Kato [1], or Goldstein [2], pp. $168 \mathrm{ff}$. , for a verification of the abstract conditions below for the case of Navier-Stokes equations. We also refer to Pazy [5], Ch. 6.3, and references therein, for an analysis of semilinear parabolic equations under similar assumptions, and to Henry [3] for further examples and theory.

On a Banach space where $\|\cdot\|$ denotes the norm as well as the induced operator norm, we consider the initial value problem

$$
y^{\prime}+A y=g(t, y), \quad y(0)=y_{0} .
$$

Here $-A$ is the generator of an analytic semigroup, with spectrum to the left of the origin:

$$
\left\|(s+A)^{-1}\right\| \leqq \frac{M}{1+|s|} \quad \text { for }|\arg s| \leqq \pi-\phi, \quad \text { with some } \phi<\frac{\pi}{2} .
$$

For the nonlinearity $\mathrm{g}$, it is assumed that there exist real numbers $\alpha$ and $\gamma<1$ such that the following local Lipschitz condition is satisfied for every $r>0$ :

$$
\|g(t, u)-g(t, v)\|_{\alpha-\gamma} \leqq L(r) \cdot\|u-v\|_{\alpha} \quad \text { for }\|u\|_{\alpha}+\|v\|_{\alpha} \leqq r, \quad 0 \leqq t \leqq T,
$$

where $\|\cdot\|_{\alpha}$ denotes the norm $\|v\|_{\alpha}=\left\|A^{\alpha} v\right\|$, defined for $v \in D\left(A^{\alpha}\right)$, the domain of $A^{\alpha}$. We note that the Stokes operator with Dirichlet boundary conditions satisfies (2) on the divergence-free subspace of $L^{2}(\Omega)^{d}$ (with a smooth bounded domain $\Omega$ in $\mathbf{R}^{d}, d=2$ or 3 ), and the nonlinearity in the Navier-Stokes equations 
satisfies (3) for any $\gamma>\frac{1}{2}$ in the case of two space dimensions, and for any $\gamma>\frac{3}{4}$ in the three-dimensional case, for both $\alpha=\gamma$ and $\alpha=\frac{1}{2}[1]$.

We consider time discretization of (1) by a linear multistep method with step size $h$, yielding discrete approximations $y_{n}, n \geqq k$, for given starting values $y_{0}, \ldots, y_{k-1}$. It is assumed that the multistep method is $A(\theta)$-stable with $\theta>\phi$ of (2), stable in a neighborhood of infinity, strongly zero-stable, and of order $p$. These are conditions (2.4) in [4], which are satisfied in particular for BDF methods. The numerical solution is compared to the solution of a perturbed equation

$$
\eta^{\prime}+A \eta=g(t, \eta)+\sigma(t)
$$

Then there is the following error bound in case that (4) has on an interval $[0, T]$ a solution which is sufficiently smooth in time.

Theorem 4' (Convergence of multistep methods for semilinear parabolic equations). Consider an $A(\theta)$-stable $k$-step method of order $p$ satisfying the conditions stated above, applied to Eqs. (1)-(3). Then the error is bounded in the norm of $D\left(A^{\alpha}\right):\|v\|_{\alpha}=\left\|A^{\alpha} v\right\| b y$

$$
\begin{aligned}
\left\|y_{n}-\eta\left(t_{n}\right)\right\|_{\alpha} \leqq & C \cdot\left(\max _{0 \leqq j \leqq k-1}\left\|y_{j}-\eta\left(t_{j}\right)\right\|_{\alpha}+h^{p} \int_{0}^{t_{n}}\left\|\eta^{(p+1)}(t)\right\|_{\alpha} d t\right. \\
& \left.+\left\|A^{-1} \sigma(0)\right\|_{\alpha}+\int_{0}^{t_{n}}\left\|A^{-1} \sigma^{\prime}(t)\right\|_{\alpha} d t\right) .
\end{aligned}
$$

This estimate holds for $t_{n}=n h \leqq T$ provided that the expression on the right-hand side is bounded by a sufficiently small constant $c$. The constants $C$ and $c$ depend only on the constants in (2) and (3), on $T$ and on $\max _{0 \leqq t \leqq T}\|\eta(t)\|_{\alpha}$. They are otherwise independent of $A$, and independent of $n$ and $h$ with $n h \leqq T$. The term involving $\sigma$ in (5) can be replaced by $\max _{0 \leqq t \leqq t_{n}}\|\sigma(t)\|$.

The proof follows closely that of Theorem 4 in [4]. In particular, it starts from the representation (cf. formula (4.13) in [4])

$$
y_{n}=h \sum_{j=k}^{n} e(n-j,-h A) g\left(t_{j}, y_{j}\right)+p_{n}
$$

where again $e(n,-h A)$ are the coefficients of the power series

$$
h \sum_{n=0}^{\infty} e(n,-h A) \zeta^{n}=(\delta(\zeta) / h+A)^{-1}, \quad|\zeta|<1,
$$

with $\delta(\zeta)$ denoting the quotient of the generating polynomials of the multistep method. The bound for the resolvent

$$
\left\|A^{\gamma}(s+A)^{-1}\right\| \leqq \text { Const } \cdot|s|^{\gamma-1}, \quad|\arg s| \leqq \pi-\phi
$$

implies via Lemma 7 of [4] (with $\mu=1-\gamma$ ) the estimate

$$
\left\|A^{\gamma} e(n,-h A)\right\| \leqq \text { Const } \cdot[(n+1) h]^{-\gamma}, \quad 0 \leqq n h \leqq T .
$$




\section{References}

1. Fujita, H., Kato, T. (1964): On the Navier-Stokes Initial Value Problem. I. Arch. Rat. Mech. Anal. 16, 269-315

2. Goldstein, J.A. (1985): Semigroups of Linear Operators and Applications. Oxford University Press, New York

3. Henry, D. (1981): Geometric Theory of Semilinear Parabolic Equations. Lecture Notes in Mathematics 840, Springer, Berlin Heidelberg New York

4. Lubich, C. (1991): On the convergence of multistep methods for nonlinear stiff differential equations. Numer. Math. 58, 839-853

5. Pazy, A. (1983): Semigroups of Linear Operators and Applications to Partial Differential Equations. Springer, New York Berlin Heidelberg 